\section{The Bystander Effect of Ultraviolet Radiation and Mediators}

\author{
Eftekhari Z. ${ }^{1.2 \odot}$, Fardid R.3,4*๑
}

\begin{abstract}
A bystander effect is biological changes in non-irradiated cells by transmitted signals from irradiated bystander cells, which causes the radiation toxic effects on the adjacent non-irradiated tissues. This phenomenon occurs by agents such as ionizing radiation, ultraviolet radiation (UVR) and chemotherapy. The bystander effect includes biological processes such as damage to DNA, cell death, chromosomal abnormalities, delay and premature mutations and micronuclei production. The most involved genes in creating this phenomenon are cyclooxygenase-2 (COX-2), inducible nitric oxide synthase (iNOS), the nuclear factor of kappa B (NFkB) and Mitogen-Activated Protein Kinases (MAPKs).

Radiation generated reactive oxygen species (ROS) can damage DNA, membranes and protein buildings. Studies have shown that Vitamin C, Hesperidin, and melatonin can reduce the number of ROS and have a protective role.

Silver nanoparticles (Ag NPs) are the most abundant nanoparticles produced and when they enter cells, they can create DNA damage. Studies have shown that combined treatment with UVR and silver nanoparticles could form $\gamma$-H2AX and 8-hydroxy-2'deoxyguanosine (8-OHdG) synergistically.

This article reviews the direct and the bystander effects of UVR on the nuclear DNA, the effect of radioprotectors and Ag NPs on these effects.
\end{abstract}

Citation: Eftekhari Z, Fardid R. The Bystander Effect of Ultraviolet Radiation and Mediators. J Biomed Phys Eng. 2020;10(1):111-118 doi: $10.31661 / \mathrm{jbpe.v0i0.956.}$

\section{Keywords}

Ultraviolet Radiation (UVR); Bystander Effect; Silver Nanoparticles; Radioprotectors; DNA Damage; Silver; Metal Nanoparticles

\section{Introduction}

\section{UVR}

The UVR creates harmful effects. Exposure to UVR creates early undesirable effects such as sunburn and long-term effects like skin cancer (malignant melanoma). UVRs are divided into three categories based on their wavelengths, including: UVRA(315-400nm), UVRB(280-315nm), UVRC(100-280nm) [1].

\section{DNA Damage by UVR}

UVRC and UVRB can express their genetic toxicity effects through direct excitation of DNA molecules. The most common UVR damage is thymine-thymine dimers and cytosine-thymine dimers. In addition to these injuries, it has been shown that exposure to UVR depends on its wavelength and provides a greater range of DNA damage such as protein-DNA crosslinking, base oxidative damage 8-hydroxy-2-deox-
${ }^{1} \mathrm{MSc}$, Department of Radiology, School of Paramedical Sciences, Shiraz University of Medical

Sciences, Shiraz, Iran

${ }^{2} \mathrm{MSc}$, Student research

committee, Shiraz

University of Medical Sci-

ences, Shiraz, Iran

${ }^{3} \mathrm{PhD}$, Department of Ra-

diology, School of Para-

medical Sciences, Shiraz

University of Medical

Sciences, Shiraz, Iran

${ }^{4} \mathrm{PhD}$, lonizing and

Non-Ionizing Radiation

Protection Research

Center (INIRPRC), School

of Paramedical Sci-

ences, Shiraz University

of Medical Sciences,

Shiraz, Iran

\section{Shiraz, Iran}

\section{R. Fardid}

*Corresponding author:

Department of Radiol-

ogy, School of Para-

medical Sciences, Shiraz

University of Medical

Sciences, Shiraz, Iran

E-mail: rfardid@sums.

ac.ir

Received: 27 May 2018

Accepted: 19 June 2018 
yguanosine (8-OHdG), single-stranded breaks and cluster damage. Especially, UVRA can cause nuclear DNA oxidation and thus indirectly DNA damage by the ROS production [2]. ROS can oxidize guanine and produce $8-\mathrm{OHdG}$, which is paired with adenine instead of cytosine. Therefore, this oxidative modification converts the $\mathrm{G} / \mathrm{C}$ pair to $\mathrm{A} / \mathrm{T}$ pair in DNA [3]. High levels of 8-OHdG have been observed in several types of cancers in humans and animals [4].

Double-strand breaks are one of the most important DNA damages. This damage is produced by external and internal cellular triggers such as ionizing radiation, genotoxic drugs and oncogenes [5]. DNA damage response (DDR) mechanisms protects the creatures against continuous genotoxic stress caused by active metabolites, environmental genotoxic agents and UVR. DDR network consists of several DNA repair mechanisms, cell cycle check points, cell senescence and apoptosis cascade signaling. Nucleotide Excision Repair (NER) is a DNA regenerative mechanism which can eliminate a lot of unstable DNA damage created by UVR [6].

\section{$\gamma-\mathrm{H} 2 \mathrm{AX}$}

Phosphorylation in serine 139 in histone $\mathrm{H} 2 \mathrm{AX}$ is called $\gamma-\mathrm{H} 2 \mathrm{AX} . \gamma-\mathrm{H} 2 \mathrm{AX}$ caused in certain types of DNA damage such as DNA double-strand breaks and plays a role in DNA repair by signaling, check points activating and organizing chromatin to increase binding DNA [7, 8]. Studies have shown that UVRC can also lead to the formation of $\gamma-\mathrm{H} 2 \mathrm{AX}$.

In 2007, Sheela Hanasoge and colleagues conducted a study on human fibroblast cells. The cells irradiated with three doses of 5,10 , $20 \mathrm{~J} / \mathrm{m}^{2} \mathrm{UVRC}$ and then $\gamma-\mathrm{H} 2 \mathrm{AX}$ production assessed by Western blot technique. The results of this study showed that control samples produced low $\gamma-\mathrm{H} 2 \mathrm{AX}$, while $\gamma-\mathrm{H} 2 \mathrm{AX}$ production increased dose-dependent manner in the samples irradiated with UVRC. Production of $\gamma-\mathrm{H} 2 \mathrm{AX}$ reached its peak two hours af- ter $5 \mathrm{~J} / \mathrm{m}^{2}$ irradiation and six hours after $10 \mathrm{~J} /$ $\mathrm{m}^{2}$ irradiation. $\gamma-\mathrm{H} 2 \mathrm{AX}$ levels had an increase in the irradiated group than the control one to 100 times in $20 \mathrm{~J} / \mathrm{m}^{2}$ UVRC irradiated cells. This increase was sustained until 12 hours. This indicates that the $\gamma$-H2AX stability duration was dependent on the UVRC dosage [8].

In 2014, Kyle Glover and colleagues conducted a study in which DDR was assessed in UVR irradiated TK6 cells. In this study, the cells were exposed to $10 \mathrm{~J} / \mathrm{m}^{2}$ UVRC radiation; then the $\gamma-\mathrm{H} 2 \mathrm{AX}$ production and content of DNA were evaluated and analyzed by flow cytometry. $\gamma-\mathrm{H} 2 \mathrm{AX}$ was low in non-irradiated cells. $10 \mathrm{~J} / \mathrm{m}^{2} \mathrm{UVRC}$ radiation increased $\gamma$-H2AX significantly in S phase cells 2 hours after irradiation; it was 56.6 percent [9].

\section{Bystander Effect}

Radiation caused the bystander effect is biological changes in non-irradiated cells, by transmitted signals from the irradiated bystander cells [10], which causes the spread of the radiation toxic effects to non-irradiated adjacent or distant tissues. This signal transmission is done either by cell direct contact or by secreted soluble factors into the culture medium [11]. In addition, ROS and reactive nitrogen species (RNS) such as cytokines are involved in mediating mechanisms in bystander effect through unknown factors [12]. Although, generally, this phenomenon is attributed to ionizing radiation, it also occurs on other stressors such as UVR, chemotherapy and Photodynamic therapy [13]. The bystander effect involves a wide range of biological processes such as DNA damage, chromosomal abnormalities, malignant transformation, cell death, apoptosis, adaptive response [5], cell viability reduction, formation of micronuclei, delay and premature mutations [12].

The genes involved in the creation of bystander effect and the inflammatory pathways are often the same. The most important of these genes are MAPKs, NFkB, iNOS and COX-2. Overexpression of these genes occurs 
by various factors and leads to inflammation and NO production as an oxidative stress increased results. UVR causes the production of macrophages to produce cytokines such as interleukin (IL)-1, IL-2, IL-8, tumor necrosis factor alpha (TNF- $\alpha$ ) and transforming growth factor beta (TGF- $\beta$ ). These factors stimulate the cytokine receptors that are located on the cell surface and facilitate gene expression. These changes are the main factors in tissue inflammation that it is irradiated directly. The observed cytokines during stimulation of gene expression of NFkB, or MAPKs genes such as extracellular signal-related kinase (ERK), JUN and P38 genes lead to COX-2 and iNOS transcription activation. COX-2 is not expressed in all tissues; in contrast, its expression level is also very low. The smallest increase in expression of this gene is clear. COX-2 is the main factor in the production of prostaglandines like PGE2, PG-I2 which causes blood vessels dilation, as well as inflammation. iNOS produces nitric oxide (NO) as well; thus, it increases the oxidative stress level. Overexpression of these genes is often associated with an increase in the COX-2. According to mentioned above, it is expected that the radiation doses cause the cytokines production, through stimulating macrophages activities, which leads to increasing COX-2 and iNOS expression in nonirradiated cells. In-vitro studies have shown that the bystander effect could have a threefold increase in COX-2, near the irradiated cells. In-vivo studies have shown that severalfold increase in the expression of these genes occurred during 72 hours after exposure [13].

A study conducted by Xiaozeng Lin and colleagues in 2017 showed that genotoxic treatments cause DDR, not only in the directly Irradiated cells, but also in the therapeutic range outside cells (bystander cells), and this phenomenon is related to the bystander effect. The study revealed that etoposide and UVR stimulate the microvesicles (MVs) production in DU145 prostate cancer cells. MVs isolated from DU145 and A431 epidermoid carcinoma cells treated with UVR, similar to those treated DU145 cells with etoposide cause ATM phosphorylation at serine 1981 (indicating activation of ATM) and H2AX histone phosphorylation at serine 139 in naïve DU145 cells. MVs neutralization derived from UVR treated cells, with annexin $\mathrm{V}$, decreased significantly with the microvesicles bystander effect activities. Etopside and UVR were known mainly for DDR induction, via ATM and RAD3 and ATM dependent pathways, respectively. In this regard, MV is probably a common source for the bystander effect caused by DNA damage. However, pretreatment of DU145 naive cells with an ATM (KU55933) inhibitor does not affect the bystander effect of isolated MVs from etoposide treated cells. This study shows that MVs are one of the bystander effect sources [14].

Widel and colleagues conducted a study in 2014 which was comparing the response of human skin fibroblast exposed cells to UVRA, UVRB, and UVRC. They used the transwellco-incubation system to investigate the created bystander effects in non-irradiated cells. The radiation effects were measured by cell survival and the apoptosis analysis. In addition, aging induction was assessed in UVR irradiated and bystander cells. Production of ROS, the Superoxide radical anions and intracellular nitric oxide and IL-6 and IL-8 secretion into the culture medium were measured and evaluated as the potential intermediary of bystander effect. The results were for 50$200 \mathrm{~J} / \mathrm{m}^{2}$ UVRC irradiated cells: the survival of directly irradiated cells was $20 \%$ and the bystander cells survival after 72 hours of exposure was $50 \%$. $200 \mathrm{~J} / \mathrm{m}^{2}$ UVRC irradiation caused apoptosis with nearly double frequency in the control group cells in 3 hours. Apoptosis frequency did not change significantly in the bystander cells. UVRC generated ROS in bystander cells significantly, especially 12 hours after the exposure. Although this increase in ROS was not observed in the directly irradiated cells, the greatest amount of produced su- 
peroxide occurred by $200 \mathrm{~J} / \mathrm{m}^{2}$ UVRC, after 3 hours to 5 times the amount of the control group and decreased quickly. Superoxide levels in bystander cells increased 2.5 times the control group cells in 2 hours after exposure. A significant increase in the level of IL-6 showed the IL- 8 to be produced by bystander cells in UVRC irradiated co-incubation systems. The IL-8 concentration was lower in the UVRC irradiated medium than in the control group cells. This reduction happened significantly in 15 minutes and 6 hours [12].

In another study conducted by Ghosh et al. in 2013, antioxidant enzymes and the bystander effect mechanisms in UVRC irradiated human melanoma A375 cells were investigated. In this study, culture medium of the UVRC irradiated human melanoma A375 cells was used to study the bystander effect. Catalase and superoxide dismutase activity reached the normal level in the treated cells 8 hours after treatment. These results indicated a strong correlation between created antioxidant activity by the bystander effect and cell sensitivity. The cell cycle suspension and antioxidant activity stimulation may cause a resistance effect or cell death, which is observed in UVRC irradiated bystander cells. This indicates the bystander effect role as a natural defense action after UVRC irradiation [15].

Dickey and colleagues conducted a study in 2009 and examined the cellular stress caused by ionizing and UVR with $\gamma$-H2AX production. In this study, target cells irradiated with non-ionizing irradiation created the bystander injury in non-target cells and this injury was assessed by measuring the amount of $\gamma-\mathrm{H} 2 \mathrm{AX}$ and 53BP1. NHF cells were used in this study. The aluminum foil was used to cover the half of the medium to investigate the bystander effect. The cells were irradiated with UVRC and incubated at different times. The least effect was a two-fold increase created 3 hours after exposure to $20 \mathrm{~J} / \mathrm{m}^{2}$. At this dose, we did not observe a significant increase in the number of $\gamma-\mathrm{H} 2 \mathrm{AX} / 53 \mathrm{BP} 1$ foci in directly irradiated cells. Adding, the medium of irradiated cells with ionizing or non-ionizing radiation was caused an increase in cytokines levels. TGF- $\beta$ and NO led to increasing in $\gamma-\mathrm{H} 2 \mathrm{AX} / 53 \mathrm{BP} 1$ foci number in the medium of normal cells as bystander cells. This increase was inhibited by the NO synthesis inhibitors, TGF- $\beta$ inhibitor antibodies and antioxidants. These results indicate that bystander cells damage is caused because they were exposed to cytokines or active components released from irradiated cells. Since this damage to the natural cells or tumor cells can cause genetic instability and through neighboring or direct contact messages may increase the risk of cancer in other cells, these results are used in oncology and radiation therapy (radiotherapy) studies [16].

\section{COX-2}

COX-2 is a critical enzyme to increase cellular proliferation, angiogenesis and tumor progression. COX-2 abnormal overexpression causes an increase in prostaglandins levels that have been seen in many cancers [17], and there is a direct association between COX-2 upregulation and increased risk of malignancies [18]. Studies have shown that UVR radiation causes overexpression of inflammatory genes such as COX-2 and this event leads to the suppression of the immune system [19].

Fardid et al. conducted a study in 2016. In this study, the dose of 3 Gy gamma radiation to the pelvis was compared with the control group; therefore it resulted in increased levels of COX-2 in the rat lung tissue $(p=0.004)$. A significant reduction (22\%) was observed in COX-2 levels in the rats pelvis that were injected with melatonin prior to irradiation with 3 Gy gamma radiation in comparison with the control group $(\mathrm{P}=0.013)$. The pelvic irradiation caused $25 \%$ COX-2 upregulation, in the non-irradiated lung tissue; this upregulation contributes to DNA oxidative damage and 8-OHdG formation. The results of this study showed that damage to DNA caused by the bystander radiation significantly increased the 
8-OHdG in comparison with the control group $(\mathrm{P}=0.013)$. Melatonin neutralizes radiation bystander effects. This means that $(\mathrm{P}<0.0005)$ 8 -OHdG levels significantly decreased in rats that melatonin received [18].

László and his colleagues conducted a study in 2009. They studied COX-2 gene expression in $30 \mathrm{~J} / \mathrm{m}^{2} \mathrm{UVRC}$ irradiated MEFS/S cells and observed that the COX-2 expression in 4-12 hours after irradiation increased 1.5 to 2 times, and TE (translation efficiency) was $2.15 \pm 0.01$ $(\mathrm{P}<0.05)$, and COX-2 synthesis significantly increased in this cell line in 4 hours [20].

\section{(Silver nanoparticles) Ag NPs}

Ag NPs with less than $100 \mathrm{~nm}$ dimensions produce abundant nanoparticles. Studies have shown that nanoparticles have greater toxicity than micro-sized particles due to their small sizes and their unique physical and chemical properties. Since the use of products containing Ag NPs has increased; concerns have increased about the Ag NPs toxicity in humans. Thus, toxicological risks of Ag NPs must be determined for safe and effective usage. Ag NPs can directly cause adverse effects. The nanoparticles can be attached directly to RNA polymerase and inhibit the RNA transcription. It can be attached to DNA and then changes its construction. It can also produce the $8-\mathrm{OHdG}$ used as a biomarker for DNA damage caused by ROS.

Zhao Xiaoxu and his colleagues in a trial examined the effects of UVR radiation and Ag NPs on the $\gamma$-H2AX production in MCF-7 cell line in 2016. In this study, they used $0-1 \mathrm{mg} / \mathrm{ml}$ Ag NPs and a 6.25-50 kJ/m² UVRA radiation. Ag NPs treatment led to $\gamma$-H2AX dose-dependent production in MCF-7 cells. Combined treatment with UVRA and Ag NPs synergistically led to forming $\gamma-\mathrm{H} 2 \mathrm{AX}$. These results showed that the Ag NPs and UVRA synergistically caused the DNA double-stranded break formation, which would eventually lead to the production of $\gamma-\mathrm{H} 2 \mathrm{AX}$. In addition, in this experiment, the cells were treated with 1 $\mathrm{mg} / \mathrm{ml} \mathrm{Ag} \mathrm{NPs} \mathrm{for} 4$ hours and then they were irradiated by $50 \mathrm{~kJ} / \mathrm{m}^{2}$ UVRA; the cells were harvested immediately after treatment and 8-OHdG formation was evaluated. As this occurred, treatment with UVRA and Ag NPs synergistically increased $8-\mathrm{OHdG}$ in the cells [21].

\section{Radioprotectors}

The ROS was produced by internal and external sources such as UVR and pollution can damage the DNA, protein buildings and membrane, and accelerate the skin aging and skin cancer progression. Vitamin $\mathrm{C}$ is a water-soluble antioxidant, which neutralizes the free radicals such as superoxide, singlet oxygen and hydroxyl radicals. Furthermore, this vitamin has antioxidant features and plays a role as a cofactor for critical enzymes in the formation of collagen, and can inhibit the elastin biosynthesis and its storage reduces. Above all, it plays a role in Vitamin E rebuilding. Vitamin $\mathrm{C}$ and $\mathrm{E}$ participate in reactions that can eliminate oxidative stress [22]. Many studies have shown that treatment with vitamin $\mathrm{C}$ before exposure to UVR radiation may play a protective role and reduce the ROS number [23]. Another radiation protector is the melatonin hormone, which is a common strategy to reduce normal tissue toxicity against ionizing radiation. Administration of this agent before and after radiotherapy can adjust the normal tissue response to radiation. Many studies have shown that melatonin, a gland pineal secreted hormone, has free radicals scavenging potential and has a strong moderating immune system [18]. Studies have also revealed that it can also reduce the UVRC damage.

Goswami and colleagues conducted a study in 2013. The results of this study showed that $265 \mathrm{~J} / \mathrm{m}^{2}$ UVRC significantly increased the apoptosis index in the irradiated splenocytes in comparison with the control group. However, in the splenocytes exposed to $250 \mathrm{Pg} / 10^{6}$ cell melatonin before incubation, a significant decrease was observed in the apoptosis index. 
Moreover, melatonin resulted in Caspase-3 activity reduction in splenocytes compared with the irradiated cells [24].

Hesperidin, as a flavonoid abundant in citrus fruits, is used widely by humans. Radiation protective effects of hesperidin have been proven in many measurement systems. In a study conducted by Fardid et al. in 2016, Hesperidin effect was evaluated on apoptosis changes and apoptotic genes target expression (Bax, Bcl-2, Bax/Bcl-2) in the rats' peripheral blood lymphocytes after gamma radiation. In this experiment a significant decrease $(\mathrm{P}<0.0001)$ was observed in lymphocyte apoptosis in animals group that had received 8 Gy radiation compared with the group that had received 2 Gy. However, in the group that received hesperidin before radiation apoptosis, an increase was observed significantly. This apoptosis increased by hesperidin administration can be attributed to Bax expression reduction, Bcl-2 expression significant decrease and ultimately $\mathrm{Bax} / \mathrm{Bcl}-2$ increase. The results of this study demonstrate that administration of 50 and $100 \mathrm{mg} / \mathrm{kg}$ Hesperidin leads to apoptotic effects by Bax expression level variation, Bcl-2 as well as the Bax/Bcl-2 [25].

A study was carried out by Shirazi et al. to investigate the effect of different doses of oral melatonin on liver tissue in 2012. The results of this study showed that whole-body radiation leads to damaging liver tissue, by increasing in MDA concentration, and decreasing glutathione (GSH) level. In the rats treated with $100,200,400 \mu \mathrm{g} / \mathrm{kg}$ melatonin before radiation, liver MDA levels decreased significantly and the GSH levels significantly increased. The results showed that oral administration of melatonin may prevent the radiation caused liver damage and this protective effect is dosedependent [26].

In another study conducted by Rezaeyan et al. in 2016, hesperidin administration effect was assessed against the tissue damage caused by gamma radiation in male rats' lung. In this experiment 32 rats were divided into four groups of eight. These rats were irradiated with 18 Gy of gamma radiation by cobalt- 60 and hesperidin was given orally with $100 \mathrm{mg} /$ $\mathrm{kg} / \mathrm{d}$ dosage seven days before exposure. The rats in each group were killed to determine SOD, GSH, MDA and histological evaluations 24 hours after radiotherapy. The results of this study revealed that in the third group (pbs + gamma ray) SOD and GSH level dropped significantly in comparison with the first group (pbs + sham irradiation). MDA increased significantly 24 hours after exposure $(\mathrm{P}=0.001$, $\mathrm{P}<0.001, \mathrm{P}=0.001)$. There was a significant difference in all parameters for the rats in the fourth group (hesperidin + gamma ray) compared to the third group $(\mathrm{P}<0.05)$. Histopathological results showed that radiation caused an increase in inflammatory lymphocytes, macrophages, and neutrophils compared with the first group 24 hours after radiotherapy $(\mathrm{P}<0.0125)$. Hesperidin oral administration before radiotherapy led to reduction of macrophages and neutrophils significantly compared with the third group $(\mathrm{P}<0.0125)$, but there was some inflammation and lymphocyte showing that there was no significant difference in comparison with the third group $(\mathrm{P}>0.0125)$. This study suggests that oral administration of hesperidin protects against gamma radiation lung injuries and rats oxidative damage; it is also likely to affect against inflammatory disorders through the free radicals scavenging ability and membrane stabilizing ability [27].

\section{Discussion}

Given the aforementioned findings, the importance of the effects of ultraviolet radiation on biological damage can be emphasized. In addition to the direct impact of ultraviolet radiation on biology and its damages, it is important to recognize the importance of the bystander effects in these radiations [28].

Certainly the following can be referred to substances or drugs that influence these processes. In some studies radiation protective effects and in some radiation sensitizers were 
The Bystander Effect of UVR and Mediators

proposed [29]. In the present study, by collecting and summarizing the existing data from previous research, some useful discussions about the bystander effect of ultraviolet radiation and what may lead to modification of this effect are presented.

\section{Conclusion}

In view of the above, a summary of the effects of UVR radiation and the influence of the presence of Ag NPs (silver nanoparticles) on the cellular environment was considered. Nowadays, the Ag NPs production and usage have increased widely. It is important that, in general, nano-sized particles are more toxic in comparison with micro-sized particles and concerns have increased in the usage of these particles. We must, therefore, identify the nanoparticles toxicity risks, for the safe and effective usage of them. Some researches demonstrate Ag NPs and UVR radiation synergistic effects on directly exposed cells, and it is likely that it also creates the synergistic effects in bystander cells. Melatonin moderating effects have been found against ionizing and non-ionizing radiation in many studies. Therefore, it seems necessary to consider this field further to provide more complete and useful results and examine the effects of other drugs.

\section{Conflict of Interest}

None

\section{References}

1. Kwon DH, Moon JD, Park WJ, Kang WY, Kim SH, Lim HM, et al. Case series of keratitis in poultry abattoir workers induced by exposure to the ultraviolet disinfection lamp. Ann Occup Environ Med. 2016;28:3. doi: 10.1186/s40557-015-00877. PubMed PMID: 26779342. PubMed PMCID: PMC4714468.

2. Fotouhi A, Hagos WW, llic M, Wojcik A, HarmsRingdahl M, De Gruijl F, et al. Analysis of mutant frequencies and mutation spectra in hMTH1 knockdown TK6 cells exposed to UV radiation. Mutat Res. 2013;751-752:8-14. doi: 10.1016/j. mrfmmm.2013.10.001. PubMed PMID: 24144844.

3. D'Orazio J, Jarrett S, Amaro-Ortiz A, Scott T. UV ra- diation and the skin. Int J Mol Sci. 2013;14:1222248. doi: 10.3390/ijms140612222. PubMed PMID: 23749111. PubMed PMCID: PMC3709783.

4. Scheuer C, Pommergaard HC, Rosenberg J, Gogenur I. Melatonin's protective effect against UV radiation: a systematic review of clinical and experimental studies. Photodermatol Photoimmunol Photomed. 2014;30:180-8. doi: 10.1111/ phpp.12080. PubMed PMID: 24164339.

5. Havaki S, Kotsinas A, Chronopoulos E, Kletsas D, Georgakilas A, Gorgoulis VG. The role of oxidative DNA damage in radiation induced bystander effect. Cancer Lett. 2015;356:43-51. doi: 10.1016/j. canlet.2014.01.023. PubMed PMID: 24530228.

6. Lans H, Marteijn JA, Schumacher B, Hoeijmakers $\mathrm{JH}$, Jansen $\mathrm{G}$, Vermeulen $\mathrm{W}$. Involvement of global genome repair, transcription coupled repair, and chromatin remodeling in UV DNA damage response changes during development. PLoS Genet. 2010;6:e1000941. doi: 10.1371/ journal.pgen.1000941. PubMed PMID: 20463888. PubMed PMCID: PMC2865526.

7. Abdel-Malek ZA, Kadekaro AL, Swope VB. Stepping up melanocytes to the challenge of UV exposure. Pigment Cell Melanoma Res. 2010;23:17186. doi: 10.1111/j.1755-148X.2010.00679.x. PubMed PMID: 20128873.

8. Hanasoge S, Ljungman M. H2AX phosphorylation after UV irradiation is triggered by DNA repair intermediates and is mediated by the ATR kinase. Carcinogenesis. 2007;28:2298-304. doi: 10.1093/ carcin/bgm157. PubMed PMID: 17615256.

9. Glover KP, Markell LK, Donner EM, Han X. Protein kinase $\mathrm{C}$-activating tumor promoters modulate the DNA damage response in UVC-irradiated TK6 cells. Toxicol Lett. 2014;229:210-9. doi: 10.1016/j. toxlet.2014.06.030. PubMed PMID: 24960060.

10. Widel M. Bystander effect induced by UV radiation; why should we be interested? Postepy Hig Med Dosw (Online). 2012;66:828-37. doi: 10.5604/17322693.1019532. PubMed PMID: 23175338.

11. Rostami A, Toossi MT, Sazgarnia A, Soleymanifard $S$. The effect of glucose-coated gold nanoparticles on radiation bystander effect induced in MCF7 and QUDB cell lines. Radiat Environ Biophys. 2016;55:461-6. doi: 10.1007/s00411-016-0669-y. PubMed PMID: 27613311.

12. Widel M, Krzywon A, Gajda K, Skonieczna M, Rzeszowska-Wolny J. Induction of bystander effects by UVA, UVB, and UVC radiation in human fibroblasts and the implication of reactive oxygen species. Free Radic Biol Med. 2014;68:278-87. doi: 
10.1016/j.freeradbiomed.2013.12.021. PubMed PMID: 24373962.

13. Najafi M, Fardid R, Hadadi G, Fardid M. The mechanisms of radiation-induced bystander effect. $J$ Biomed Phys Eng. 2014;4:163-72. PubMed PMID: 25599062. PubMed PMCID: PMC4289523.

14. Lin X, Wei F, Major P, Al-Nedawi K, Al Saleh HA, Tang D. Microvesicles Contribute to the Bystander Effect of DNA Damage. Int J Mol Sci. 2017;18: 788. doi: 10.3390/ijms18040788. PubMed PMID: 28387728. PubMed PMCID: PMC5412372.

15. Ghosh R, Guha D, Bhowmik S, Karmakar S. Antioxidant enzymes and the mechanism of the bystander effect induced by ultraviolet $C$ irradiation of A375 human melanoma cells. Mutat Res. 2013;757:83-90. doi: 10.1016/j.mrgent0x.2013.06.022. PubMed PMID: 23845763.

16. Dickey JS, Baird BJ, Redon CE, Sokolov MV, Sedelnikova OA, Bonner WM. Intercellular communication of cellular stress monitored by gamma-H2AX induction. Carcinogenesis. 2009;30:1686-95. doi: 10.1093/carcin/bgp192. PubMed PMID: 19651821. PubMed PMCID: PMC2757548.

17. Byun S, Lee KW, Jung SK, Lee EJ, Hwang MK, $\mathrm{Lim} S \mathrm{SH}$, et al. Luteolin inhibits protein kinase $\mathrm{C}$ (epsilon) and c-Src activities and UVB-induced skin cancer. Cancer Res. 2010;70:2415-23. doi: 10.1158/0008-5472.CAN-09-4093. PubMed PMID: 20215519.

18. Fardid R, Salajegheh A, Mosleh-Shirazi MA, Sharifzadeh S, Okhovat MA, Najafi M, et al. Melatonin ameliorates the production of COX-2, iNOS, and the formation of 8-OHdG in non-targeted lung tissue after pelvic irradiation. Cell Journal (Yakhteh). 2017;19:324-331.

19. Katiyar SK. Dietary proanthocyanidins inhibit UV radiation-induced skin tumor development through functional activation of the immune system. Mol Nutr Food Res. 2016;60:1374-82. doi: 10.1002/ mnfr.201501026. PubMed PMID: 26991736. PubMed PMCID: PMC4899090.

20. László CF, Fayad S, Carpenter OL, George KS, Lu W, Saad AA, Wu S. The role of translational regulation in ultraviolet $C$ light-induced cyclooxygenase-2 expression. Life Sci. 2009;85:70-6. doi: 10.1016/j.Ifs.2009.04.018.
21. Zhao X, Takabayashi F, Ibuki Y. Coexposure to silver nanoparticles and ultraviolet A synergistically enhances the phosphorylation of histone H2AX. J Photochem Photobiol B. 2016;162:213-22. doi: 10.1016/j.jphotobiol.2016.06.046. PubMed PMID: 27383448.

22. Chen L, Hu JY, Wang SQ. The role of antioxidants in photoprotection: a critical review. $J \mathrm{Am}$ Acad Dermatol. 2012;67:1013-24. doi: 10.1016/j. jaad.2012.02.009. PubMed PMID: 22406231.

23. Poljsak B, Dahmane R. Free radicals and extrinsic skin aging. Dermatol Res Pract. 2012;2012:135206. doi: 10.1155/2012/135206. PubMed PMID: 22505880. PubMed PMCID: PMC3299230.

24. Goswami S, Sharma S, Haldar C. The oxidative damages caused by ultraviolet radiation type C (UVC) to a tropical rodent Funambulus pennanti: role of melatonin. $J$ Photochem Photobiol B. 2013;125:19-25. doi: 10.1016/j.jphotobiol.2013.04.008. PubMed PMID: 23708058.

25. Fardid R, Ghorbani Z, Haddadi G, Behzad-Behbahani A, Arabsolghar R, Kazemi E, et al. Effects of Hesperidin as a Radio-protector on Apoptosis in Rat Peripheral Blood Lymphocytes after Gamma Radiation. J Biomed Phys Eng. 2016;6:21728. PubMed PMID: 28144590. PubMed PMCID: PMC5219572.

26. Shirazi A, Fardid R, Mihandoost E. Protective effect of low dose melatonin on radiation-induced damage to rat liver. Journal of Biomedical Physics and Engineering. 2012;2(2): 66-71.

27. Rezaeyan A, Fardid R, Haddadi GH, Takhshid MA, Hosseinzadeh M, Najafi M, et al. Evaluating Radioprotective Effect of Hesperidin on Acute Radiation Damage in the Lung Tissue of Rats. J Biomed Phys Eng. 2016;6:165-74. PubMed PMID: 27853724. PubMed PMCID: PMC5106549.

28. Eftekhari-Kenzerki Z, Fardid R, Behzad-Behbahani A. Impact of silver nanoparticles on the ultraviolet radiation direct and bystander effects on TK6 cell line. J Med Phys. 2019;44:118-25. doi: 10.4103/ jmp.JMP_111_18.

29. Zare T, Fardid R, Naderi S. Synergetic effect of silver nanoparticles and UVC irradiation on H2AX gene expression in TK6 cells. Cell J. 2019; 21(2): 204-209. doi: 10.22074/cellj.2019.5898. 